\title{
Nuclear respiratory factor 1 overexpression attenuates anti-benzopyrene-7,8-diol-9,10-epoxide-induced S-phase arrest of bronchial epithelial cells
}

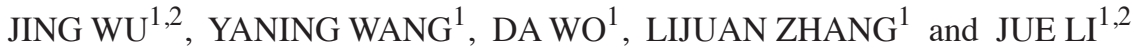 \\ ${ }^{1}$ Division of Preventive Medicine; ${ }^{2}$ Heart, Lung and Blood Vessel Center, \\ Tongji University School of Medicine, Shanghai 200092, P.R. China
}

Received April 21, 2015; Accepted January 11, 2016

DOI: $10.3892 / \mathrm{mmr} .2016 .5065$

\begin{abstract}
Nuclear respiratory factor 1 (NRF-1) has important roles in the regulation of several key metabolic genes required for cellular growth and respiration. A previous study by our group indicated that NRF-1 is involved in mitochondrial dysfunction induced by the environmental pollutant benzo[a] pyrene in the $16 \mathrm{HBE}$ human bronchial epithelial cell line. In the present study, it was observed that its genotoxic metabolite, anti-benzopyrene-7,8-diol-9,10-epoxide (BPDE), triggered cell cycle arrest in $\mathrm{S}$-phase in $16 \mathrm{HBE}$ cells by activating ataxia-telangiectasia (ATM)/checkpoint kinase (Chk)2 and ATM and Rad3 related (ATR)/Chk1 signaling pathways. NRF-1 expression was suppressed by BPDE after treatment for $6 \mathrm{~h}$. Flow cytometric analysis revealed that NRF-1 overexpression attenuated cell cycle arrest in S-phase induced by BPDE. In line with this result, DNA-damage checkpoints were activated following NRF-1 overexpression, as demonstrated by increased phosphorylation of ATM, Chk2 and $\gamma \mathrm{H} 2 \mathrm{AX}$, but not ATR and Chk1, according to western blot analysis. It was therefore indicated that NRF-1 overexpression attenuated BPDE-induced S-phase arrest via the ATM/Chk2 signaling pathway.
\end{abstract}

Correspondence to: Professor Jue Li or Dr Lijuan Zhang, Division of Preventive Medicine, Tongji University School of Medicine, 1239 Siping Road, Shanghai 200092, P.R. China

E-mail: allydata88@126.com

E-mail:wujingclose@hotmail.com

Abbreviations: BPDE, anti-benzopyrene-7,8-diol-9,10-epoxide; NRF-1, nuclear respiratory factor 1; ATM, ataxia-telangiectasia; ATR, ATM and Rad3 related; Chk1/2, checkpoint kinase 1/2; $\gamma \mathrm{H} 2 \mathrm{AX}$, gamma histone variant $\mathrm{H} 2 \mathrm{AX}$

Key words: nuclear respiratory factor 1, anti-benzopyrene-7, 8-diol-9, 10-epoxide, cell cycle, DNA damage checkpoint

\section{Introduction}

Nuclear respiratory factor-1 (NRF-1), which induces nuclear-encoded mitochondrial genes, is a master transcription factor for mitochondrial function and biogenesis (1). In proliferating mammalian cells, NRF-1 is phosphorylated at multiple serine residues within a concise $\mathrm{NH}_{2}$-terminal domain that enhances its DNA-binding affinity and trans-activation function (2). Bioinformatics analyses suggested that NRF-1 has an integrative role in the transcriptional network controlling cell cycle regulatory genes (3). In addition, NRF-1 has been identified as a co-regulator of a large subset of E2F-responsive genes (4). Growing evidence suggested that NRF-1 was associated with the regulation of neurite outgrowth $(5,6)$. This led to the hypothesis that NRF-1 may have roles in the regulation of the cell cycle.

Mitochondria are eukaryotic organelles that contain their own genetic system known as the mitochondrial DNA (mtDNA) genome. Nucleo-mitochondrial interactions commonly depend on the crosstalk between nuclear transcription factors and the co-activators of PGC-1 family members (7). NRF-1 promotes the expression of Tfam and two TFB isoforms (TFB1M or TFB2M) that are essential for mtDNA maintenance (8). Veatch et al (9) observed that the loss of mtDNA led to a reduction in the cell growth rate as well as cell cycle arrest. An in vivo study showed that targeted disruption of NRF-1 results in early embryonic lethality and a marked reduction in the amount of mtDNA (10). Therefore, it is indicated that NRF-1 may serve as a pivotal regulator of cell cycle progression and mitochondrial DNA stability. However, whether NRF-1 promotes cell proliferation has remained to be fully demonstrated.

Benzo[a]pyrene $(\mathrm{BaP})$ is an ubiquitous environmental pollutant with known carcinogenic properties. Oxidation of $\mathrm{BaP}$ by human cytochrome P-450 enzymes forms a more potent genotoxic derivative named BPDE (11). The underlying molecular mechanism of its carcinogenic effects has been shown to be associated with the induction of DNA damage. Exposure of cultured cells to BPDE has been demonstrated to elicit cell cycle arrest in G1 and S-phases in either a p53-dependent or -independent manner $(12,13)$. A previous study by our group has documented that BPDE-induced apoptosis proceeded via 
the mitochondrial-mediated pathway (14). Thus far, the mechanisms leading to DNA damage, transcriptional regulation and mitochondrial dysfunction following BPDE exposure have largely remained elusive. Given the potential role of NRF-1 in regulating cell growth and mitochondrial biogenesis, the present study hypothesized that NRF-1 may have crucial roles in BPDE-induced cell cycle arrest. To test this hypothesis, the effects of NRF-1 overexpression on cell cycle arrest and the activity of cell checkpoint pathways induced by BPDE were investigated.

\section{Materials and methods}

Cell culture and reagents. The $16 \mathrm{HBE}$ human bronchial epithelial cell line was purchased from the Tumor Marker Research Center (Beijing, China). Cells were cultured in RPMI-1640 media supplemented with $10 \%$ fetal bovine serum (Gibco; Thermo Fisher Scientific, Inc., Waltham, MA, USA) at $37^{\circ} \mathrm{C}$ in a humidified atmosphere with $5 \% \mathrm{CO}_{2}$. BPDE was purchased from the National Cancer Institute (NCI, Rockville, MD, USA). Phenylmethanesulfonyl fluoride (PMSF) and phosphatase inhibitor cocktail were from Sigma-Aldrich (St. Louis, MO, USA). Lipofectamine ${ }^{\mathrm{TM}} 2000$ was from Invitrogen (Thermo Fisher Scientific,Inc.). Mouse monoclonal anti-NRF-1 antibody (cat. no. sc-101102), goat anti-rabbit IgG-HRP (cat. no. sc-2004) and goat anti-mouse IgG secondary antibodies (cat. no. sc-2039) were purchased from Santa Cruz Biotechnology, Inc. (Dallas, TX, USA). Rabbit polyclonal anti-p-Ser428-ATR (cat. no. 2853), rabbit polyclonal anti-p-Ser1981-ATM (cat. no. 5883), rabbit polyclonal anti-p-Ser1524-BRCA1 (cat. no. 9009), rabbit monoclonal anti-p-Thr68-Chk2 (cat. no. 2197), rabbit monoclonal anti-p-Ser345-Chk1 (cat. no. 2348), mouse monoclonal anti- $\beta$-actin (cat. no. 3700 ) and rabbit monoclonal anti-GAPDH (cat. no. 5174) were obtained from Cell Signaling Technology, Inc. (Danvers, MA, USA). A Cell Cycle Detection kit was obtained from KeyGen Biotech Co, Ltd. (Nanjing, China). A Cell Counting Kit-8 (CCK-8) was purchased from Dojindo Molecular Technologies (Kumamoto, Japan) and a bicinchoninic acid (BCA) protein assay kit was from Pierce Biotechnology, Inc. (Rockford, IL, USA).

Cytotoxicity assays. Cells were plated in 96-well plates and treated with BPDE at $0,0.25,0.5$ or $1 \mu \mathrm{M}$ for 3,6 or $12 \mathrm{~h}$ under low-serum ( $0.5 \%$ FBS) conditions. Cytotoxicity was assessed using the CCK- 8 assay according to the manufacturer's instructions. In brief, $10 \mathrm{ml}$ CCK- 8 solution was added to the cell cultures, followed by incubation for $1.5 \mathrm{~h}$ in the dark. The absorbance was measured at $450 \mathrm{~nm}$ using an mQuant microplate reader (Biotek, Winooski, VT, USA). For counting of viable cells, cultures treated as stated above were harvested, stained with Trypan Blue and counted with a hemocytometer ( $0.1 \mathrm{~mm}$; Beyotime Institute of Biotechnology, Haimen, China).

Cell cycle analysis. The cell cycle was assessed using the Cell Cycle Detection kit (KeyGen Biotech Co, Ltd.) according to the manufacturer's instructions. Following treatment with BPDE $(0,0.25$ or $0.5 \mu \mathrm{M}$ for 6 or $12 \mathrm{~h})$, cells were harvested using $0.05 \%(\mathrm{w} / \mathrm{v})$ trypsin (Gibco; Thermo Fisher Scientific, Inc.) in phosphate-buffered saline (PBS) containing $0.02 \%(\mathrm{w} / \mathrm{v})$ sodium ethylenediaminetetraacetate. Cells were fixed in $70 \%$ ethanol for $2 \mathrm{~h}$, followed by two washes with PBS and incubation with RNase A $(100 \mu \mathrm{g} / \mathrm{ml})$ at $37^{\circ} \mathrm{C}$ for $30 \mathrm{~min}$. Propidium iodide was then used to stain the cells in the dark for $30 \mathrm{~min}$. The samples were analyzed using a FACScan flow cytometer (BD Biosciences, San Jose, CA, USA). A total of 10,000 cells were counted for each sample. Determination of the cell population and quantification of the cells were performed using the CellQuest software (version 5.1; BD Biosciences).

Protein extraction and western blot analysis. Following treatment, cells were washed with PBS twice and then lysed in cell lysis buffer containing $1 \%$ PMSF and $1 \%$ protein protease inhibitor cocktail (Pierce Biotechnology, Inc.) for $30 \mathrm{~min}$ on ice. Protein concentrations of extracts (nuclear or cytoplasmic) were determined using the BCA protein assay kit. Equal quantities $(20-30 \mu \mathrm{g})$ of protein were separated on $6-12 \%$ gradient sodium dodecyl sulfate polyacrylamide gels (JRDun Biotechnology Co., Ltd., Shanghai, China) and then transferred onto polyvinylidene difluoride membranes (Merck-Millipore, Bedford, MA, USA). Membranes were blocked for $1 \mathrm{~h}$ with 5\% non-fat milk or $3 \%$ bovine serum albumin (Sigma-Aldrich) in Tris-buffered saline (137 mM sodium chloride, $20 \mathrm{mM}$ Tris, $\mathrm{pH}$ 7.6) containing $0.05 \%$ Tween-20 (TBST) at room temperature and incubated with primary antibodies overnight at $4^{\circ} \mathrm{C}$. The dilution ratio of the primary antibodies was $1: 1,000$, with the exception of anti-GAPDH and $\beta$-actin antibody $(1: 5,000)$. Membranes were then washed with TBST three times per $10 \mathrm{~min}$ and incubated with horseradish peroxidase-conjugated secondary antibody $(1: 5,000)$ for $1 \mathrm{~h}$ at room temperature. Finally, the immunoreactive bands were detected using an enhanced chemiluminescence kit (Pierce, Biotechnology, Inc.). LabWorks Image Acquisition (UVGL-58; UVP, Upland, CA, USA) was used to capture images.

Plasmids and transient transfection. A fragment encoding the NRF-1 protein (GenBank accession no, M_005011) was amplified by polymerase chain reaction from human NRF-1 expression vector. The PCR reaction was set up in a total volume of $20 \mu \mathrm{l}$ and contained 5X Taq buffer (Dalian Sinobio Chemistry Co., Ltd., Dalian, China), 2.5 mM dNTP (Takara Bio, Inc., Otsu, Japan), $10 \mu \mathrm{M}$ primers (Generay Biotech Co., Shanghai, China), Taq polymerase (Dalian Sinobio Chemistry Co., Ltd.), $10 \mathrm{ng} / \mu \mathrm{l}$ template and double distilled water. The sequences of the primers were as follows: Forward, 5'-TCCGCTCGAGATGGAGGAACACGGAGTG AC-3' and reverse, 5'-ATGGGGTACCGACTGTTCCAATGT CACCACCTC-3'. Amplification was performed using an Applied Biosystems 2720 ThermalCycler(Applied Biosystems, Foster City, CA, USA). Following initial denaturation at $94^{\circ} \mathrm{C}$ for $5 \mathrm{~min}, 30$ cycles of amplification were performed, including $94^{\circ} \mathrm{C}$ for $30 \mathrm{sec}$ denaturation time, $55^{\circ} \mathrm{C}$ for $30 \mathrm{sec}$ annealing, $72^{\circ} \mathrm{C}$ for $2 \mathrm{~min}$ extension, and a final extension of $72^{\circ} \mathrm{C}$ for $10 \mathrm{~min}$. The PCR product was cut with EcoRI and KpnI (Shanghai Genechem Co., Ltd., Shanghai, China). The NRF-1 cDNA fragments were inserted into the GV141 vector (CMV-MCS-3FLAG-SV40-Neomycin; GeneChem, Shanghai, China), and the pcDNA-NRF-1 plasmid was obtained. $16 \mathrm{HBE}$ cells were each plated in $35 \mathrm{~mm}$ dishes at a density of $2 \times 10^{5}$ cells/dish. Cells were transfected $24 \mathrm{~h}$ post-plating with 
A

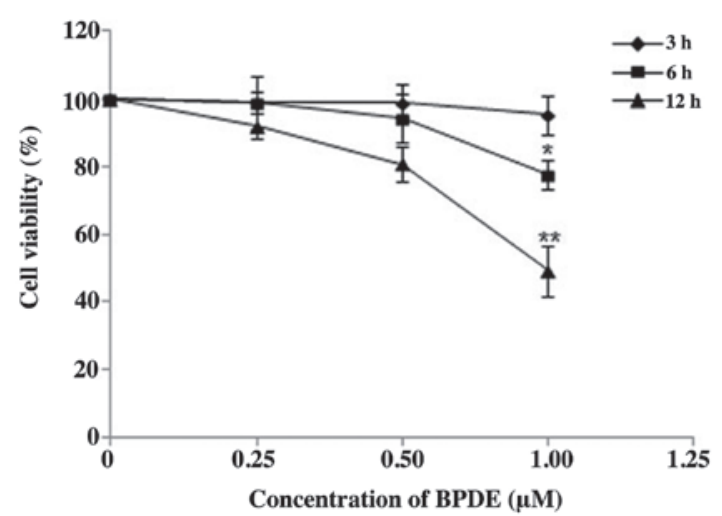

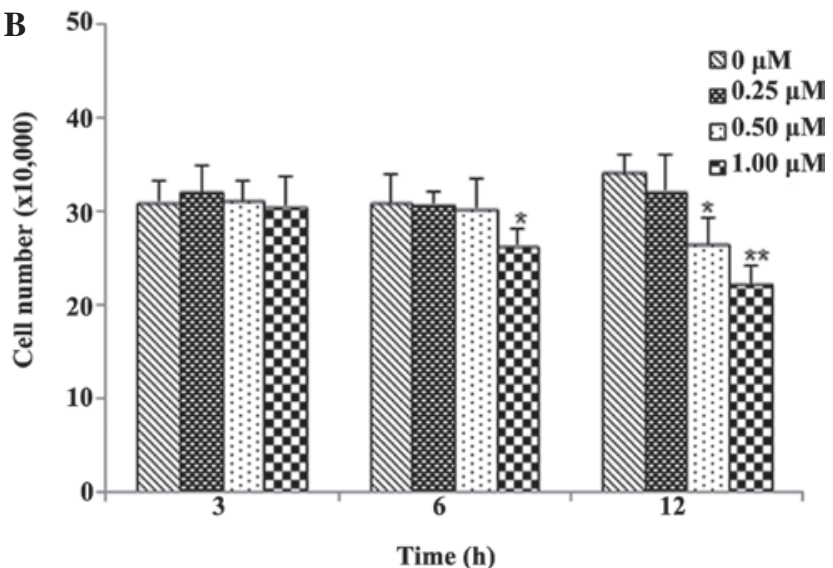

Figure 1. Effect of BPDE on the viability and number of 16HBE cells. (A) Cell viability was measured using a Cell Counting Kit 8 following treatment with BPDE at various concentrations for 3,6 and $12 \mathrm{~h}$. Values are expressed as a percentage of the control. (B) The cell number was monitored by the ability to exclude trypan blue. Values are expressed as the mean \pm standard deviation. ${ }^{*} \mathrm{P}<0.05,{ }^{* *} \mathrm{P}<0.01$ vs. untreated cells. The experiments were repeated at least three times independently. BPDE, anti-benzopyrene-7,8-diol-9,10-epoxide.

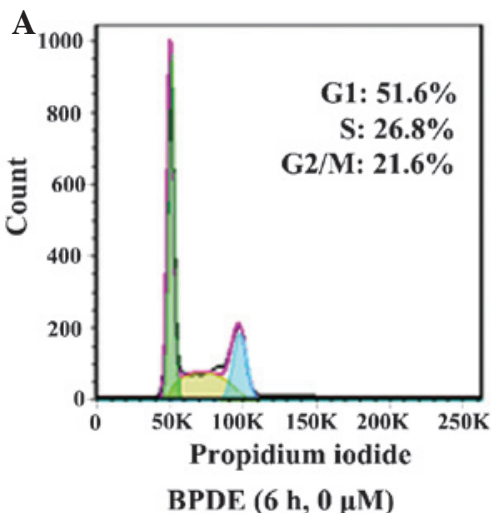

D

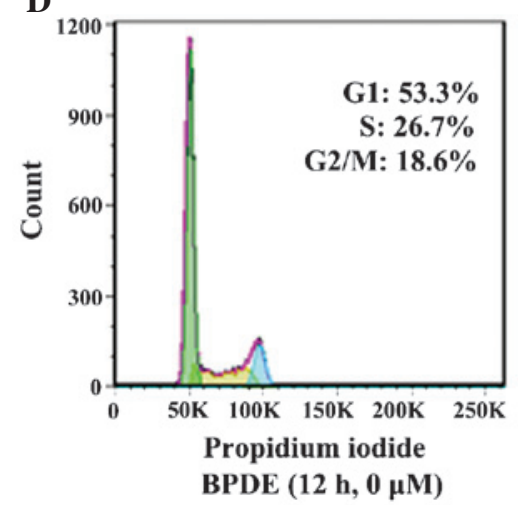

B

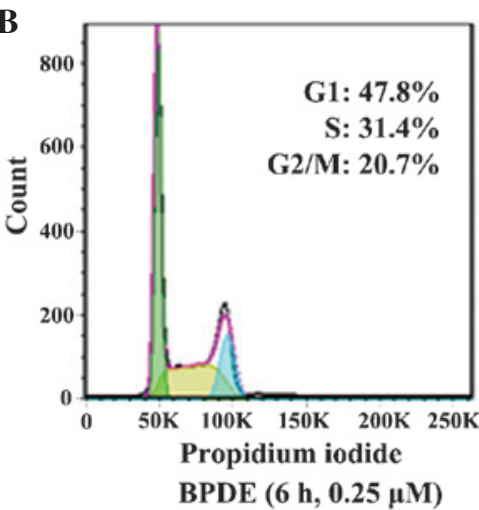

$\mathbf{E}$

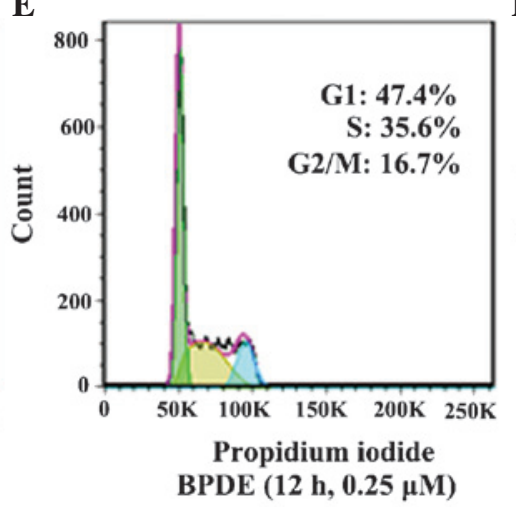

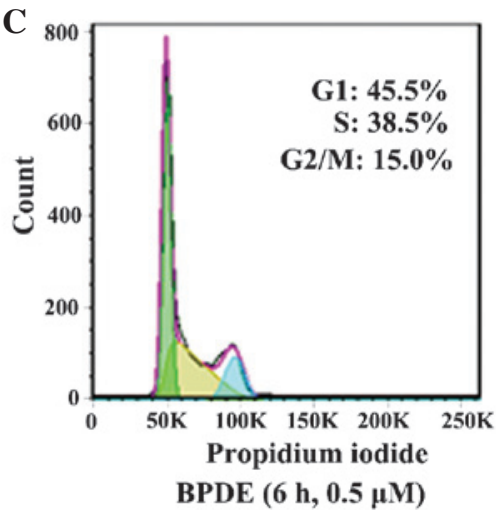

F

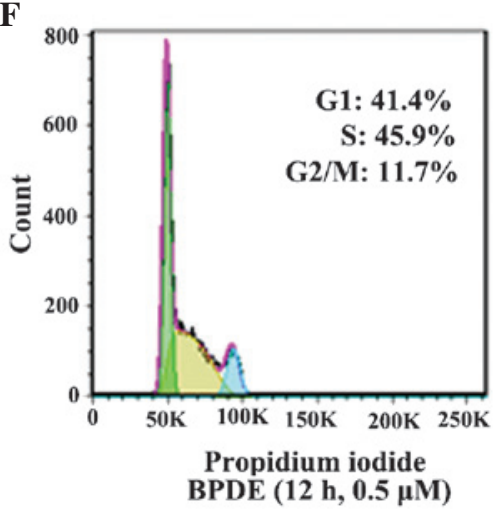

Figure 2. Effects of BPDE on the cell cycle distribution of 16HBE cells. (A-F) Cell cycle distribution in different conditions. Cell cycle distribution was determined using flow cytometry. After BPDE treatment for 6 and $12 \mathrm{~h}$ under low serum $(0.5 \%)$ conditions, cells were harvested and stained with propidium iodide. BPDE, anti-benzopyrene-7,8-diol-9,10-epoxide.

either $2 \mu \mathrm{g}$ of the NRF-1 plasmid or a control vector, using Lipofectamine $^{\mathrm{TM}} 2000$ (Invitrogen; Thermo Fisher Scientific, Inc.) at a 1:3 ratio. Following transfection for $24 \mathrm{~h}$, cells were treated with BPDE for $6 \mathrm{~h}$ or $12 \mathrm{~h}$, and cells were subsequently subjected to cell cycle and western blot analysis according to the abovementioned procedures.

Statistical analysis. Statistical analysis was performed using SPSS 14.0 (SPSS, Inc., Chicago, IL, USA). Values are expressed as the mean \pm standard deviation. Experimental data were analyzed using one-way analysis of variance (ANOVA) followed by Tukey's multiple range test for significant differences. $\mathrm{P}<0.05$ was considered to indicate a statistically significant difference between values.

\section{Results}

$B P D E$ reduces the proliferation of bronchial epithelial cells. To assess the effects of BPDE on cell proliferation, 16HBE cells were treated with various concentrations of BPDE for various 
Table I. Percentage of cells in different cell cycle phases after BPDE treatment.

\begin{tabular}{|c|c|c|c|c|c|c|}
\hline \multirow[b]{2}{*}{ Cell cycle $(\%)$} & \multicolumn{3}{|c|}{ BPDE (6 h) } & \multicolumn{3}{|c|}{ BPDE (12 h) } \\
\hline & $0 \mu \mathrm{M}$ & $0.25 \mu \mathrm{M}$ & $0.5 \mu \mathrm{M}$ & $0 \mu \mathrm{M}$ & $0.25 \mu \mathrm{M}$ & $0.5 \mu \mathrm{M}$ \\
\hline G1 & $51.4 \pm 3.6$ & $46.1 \pm 2.9$ & $45.1 \pm 3.2$ & $53.4 \pm 4.2$ & $48.7 \pm 3.5$ & $42.6 \pm 3.5$ \\
\hline $\mathrm{S}$ & $26.4 \pm 4.1$ & $30.9 \pm 4.3$ & $38.2 \pm 2.4$ & $26.7 \pm 3.1$ & $34.8 \pm 2.1$ & $45.1 \pm 2.6$ \\
\hline $\mathrm{G} 2 / \mathrm{M}$ & $21.8 \pm 3.7$ & $22.2 \pm 2.6$ & $15.5 \pm 1.9$ & $18.8 \pm 2.8$ & $15.7 \pm 3.2$ & $11.1 \pm 2.3$ \\
\hline
\end{tabular}

BPDE, anti-benzopyrene-7,8-diol-9,10-epoxide.

Table II. Percentage of cells in different cell cycle phases in response to BPDE treatment and NRF-1 overexpression.

\begin{tabular}{lcccr}
\hline & \multicolumn{2}{c}{ Control } & & BPDE \\
\cline { 2 - 5 } Cell cycle $(\%)$ & Vector & pcDNA-NRF-1 & Vector & pcDNA-NRF-1 \\
\hline G1 & $56.8 \pm 3.1$ & $55.6 \pm 1.8$ & $46.9 \pm 3.7$ & $53.4 \pm 1.9$ \\
S & $26.1 \pm 1.6$ & $26.5 \pm 2.9$ & $40.7 \pm 2.0$ & $28.3 \pm 2.4$ \\
G2/M & $14.4 \pm 1.7$ & $16.0 \pm 2.1$ & $11.1 \pm 1.6$ & $17.0 \pm 2.6$ \\
\hline
\end{tabular}

NRF-1, nuclear respiratory factor 1; BPDE, anti-benzopyrene-7,8-diol-9,10-epoxide.

A

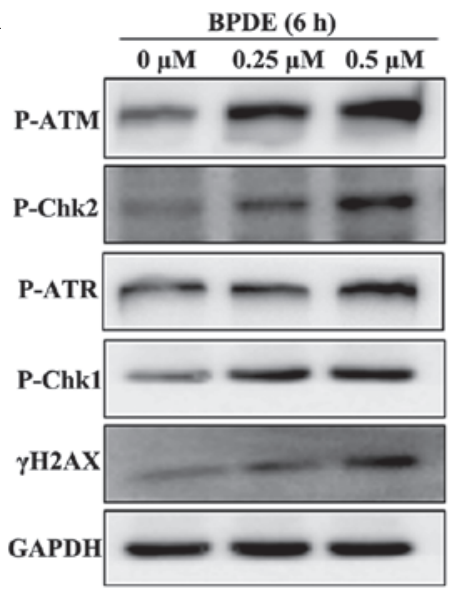

B

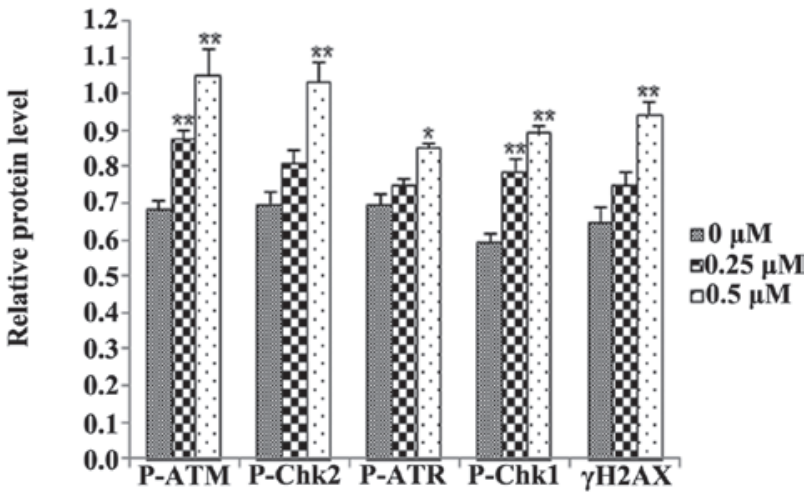

Figure 3. DNA damage checkpoints are activated by BPDE. Following BPDE treatment for $6 \mathrm{~h}$ under low serum $(0.5 \%)$ conditions, cells were harvested for western blot analysis. (A) Representative western blot; (B) Quantification of protein levels by densitometric analysis. GAPDH was used as a loading control. Data are expressed as percentage of control. Values are expressed as the mean \pm standard deviation. ${ }^{*} \mathrm{P}<0.05,{ }^{* * *} \mathrm{P}<0.01$ vs. untreated cells. The experiments were repeated at least three times independently. P-Chk2, phosphorylated checkpoint kinase $2 ; \gamma \mathrm{H} 2 \mathrm{AX}$, gamma histone variant $\mathrm{H} 2 \mathrm{AX}$; ATM, ataxia-telangiectasia; ATR, ATM and Rad3 related; BPDE, anti-benzopyrene-7,8-diol-9,10-epoxide; GAPDH, glyceraldehyde-3-phosphate dehydrogenase.

A
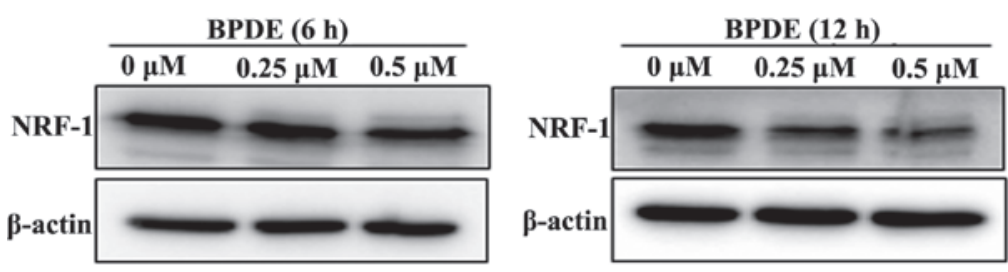

B

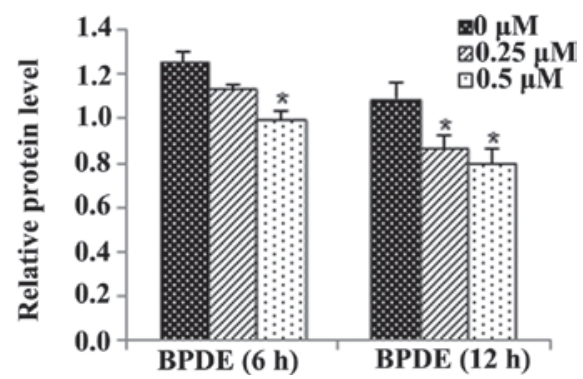

Figure 4. NRF-1 expression levels are downregulated in response to BPDE. (A) NRF-1 protein levels were analyzed by western blotting after BPDE treatment for the indicated times. (B) The relative protein levels of NRF-1 in the control and BPDE groups were determined by densitometric analysis. Values are expressed as the mean \pm standard deviation. " $\mathrm{P}<0.05$ vs. untreated cells. BPDE, anti-benzopyrene-7,8-diol-9,10-epoxide; NRF-1, nuclear respiratory factor 1 . 

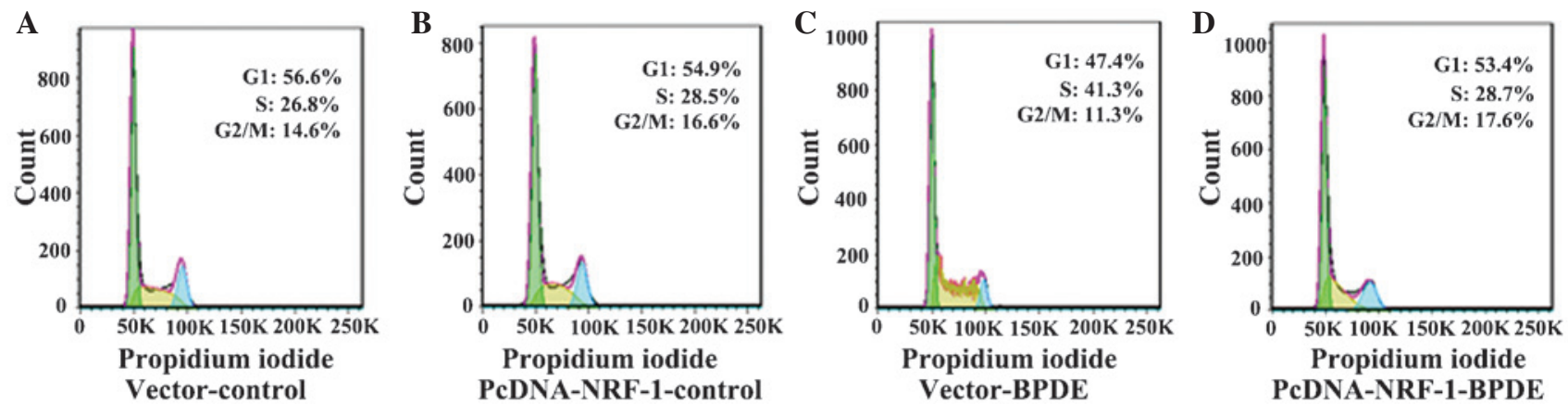

Figure 5. NRF-1 overexpression attenuates BPDE-induced S phase arrest. 16HBE cells were transfected with (A) vector or (B) PcDNA-NRF-1 for 24 h and (C and D) a proportion of these cells was subsequently treated with BPDE for $6 \mathrm{~h}$. The cell cycle distribution was analyzed by flow cytometry. NRF-1, nuclear respiratory factor 1; BPDE, anti-benzopyrene-7,8-diol-9,10-epoxide.

A

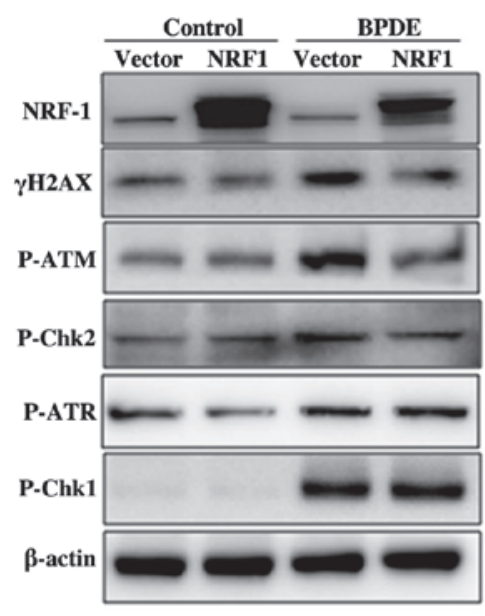

B

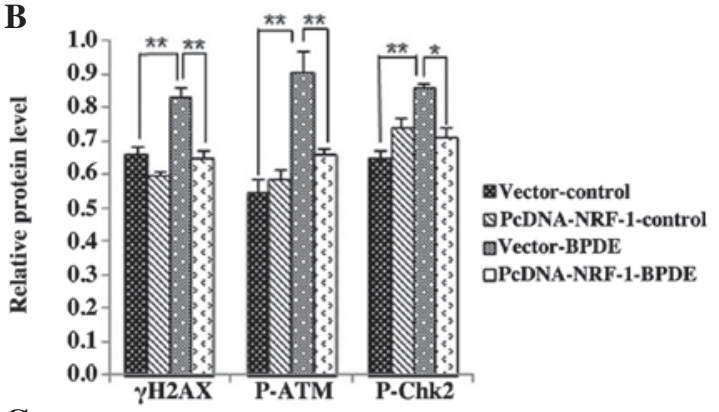

C

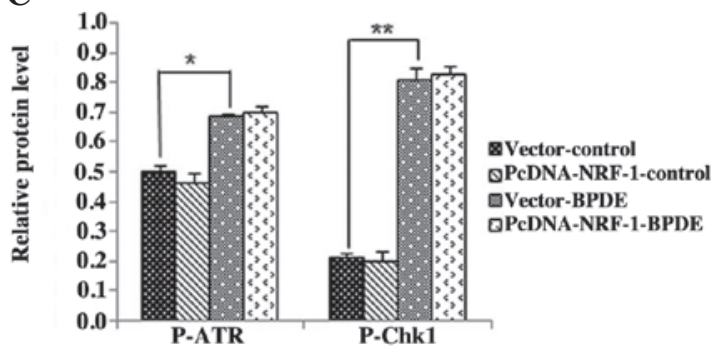

Figure 6. Changes of checkpoint activation in response to BPDE and NRF-1 overexpression. (A) Blots showing protein expression levels of $\gamma$ H2AX, P-ATM, P-Chk2, P-ATR and P-Chk1 in response to BPDE exposure and NRF-1 overexpression. (B and C) Relative protein levels in control and BPDE groups. Values are expressed as the mean \pm standard deviation. ${ }^{*} \mathrm{P}<0.05,{ }^{* *} \mathrm{P}<0.01$ vs. control. P-Chk2, phosphorylated checkpoint kinase $2 ; \gamma \mathrm{H} 2 \mathrm{AX}$, gamma histone variant H2AX; ATM, ataxia-telangiectasia; ATR, ATM and Rad3 related; BPDE, anti-benzopyrene-7,8-diol-9,10-epoxide; GAPDH, glyceraldehyde-3-phosphate dehydrogenase.

durations under low-serum $(0.5 \%)$ conditions and subjected to the CCK-8 assay. A time- and concentration-dependent inhibition of cell growth was observed following treatment with BPDE ( $\mathrm{P}<0.05,1.0$ vs. $0 \mu \mathrm{M}$ at $12 \mathrm{~h}$ ) (Fig. 1A). When the concentration of BPDE exceeded $0.5 \mu \mathrm{M}$, cell proliferation was significantly inhibited (Fig. 1B).

BPDE induces $S$-phase arrest of bronchial epithelial cells. The effects of BPDE on the cell cycle distribution of $16 \mathrm{HBE}$ cells was determined by flow cytometry. As shown in Fig. 2A-F, treatment with 0.25 or $0.5 \mu \mathrm{M}$ BPDE for 6 or $12 \mathrm{~h}$ significantly increased the proportion of cells in S phase. BPDE $(0.5 \mu \mathrm{M})$ increased the S-phase population by $11.3 \pm 2.1$ and $20.7 \pm 4.5 \%$, respectively, at 6 and $12 \mathrm{~h}(\mathrm{P}<0.01)$ (Table I). These results suggested that BPDE induced S-phase arrest in 16HBE cells.

BPDE induces DNA damage checkpoint activation. BPDE treatment upregulated the levels of p-ATR (Ser428) and its downstream mediator p-Chk1 (Fig. $3 \mathrm{~A}$ and $\mathrm{B}$ ). In order to explore whether ATM/Chk2 signaling was also involved in BPDE-induced S-phase arrest, the levels of p-ATM (Ser1981) and p-Chk2 (Thr68) were also determined. The results revealed that these checkpoint proteins were increased in a dose-dependent manner following BPDE treatment (Fig. 3A and $\mathrm{B}$ ). In addition, the levels of $\gamma \mathrm{H} 2 \mathrm{AX}$ were increased following BPDE (Fig. 3B). These findings indicated that ATM/Chk2 and ATR/Chk1 cascades were involved in DNA damage checkpoint signaling in $16 \mathrm{HBE}$ cells initiated by BPDE treatment.

The expression levels of NRF-1 in response to BPDE induced $S$-phase arrest. A previous study by our group observed that the mRNA and protein levels of NRF-1 were decreased following treatment with $16 \mu \mathrm{M} \mathrm{BaP}$ for $48 \mathrm{~h}$ (14). To investigate whether endogenous NRF-1 levels were altered in response to BPDE, $16 \mathrm{HBE}$ cells were treated with BPDE at concentrations of 0 , 0.25 or $0.5 \mu \mathrm{M}$ for 6 or $12 \mathrm{~h}$. The total protein levels of NRF-1 were significantly reduced following treatment with $0.25 \mu \mathrm{M}$ 
BPDE for $12 \mathrm{~h}$ as well as with $0.5 \mu \mathrm{M}$ BPDE for 6 and $12 \mathrm{~h}$ $(\mathrm{P}<0.05)$ (Fig. 4A and B).

Overexpression of NRF attenuates $S$-phase arrest and cell cycle checkpoint activation induced by BPDE. To examine whether NRF-1 was involved in the regulation of the cell cycle arrest induced by BPDE, the effects of NRF-1 overexpression on the cell cycle distribution of $16 \mathrm{HBE}$ cells was assessed. No significant differences in cell cycle distribution were observed between cells transiently transfected with a plasmid encoding NRF-1 and those transfected with control (empty) vector (Fig. 5A and B). Cells transfected with the control vector were susceptible to genotoxic stress induced by BPDE, as indicated by a significantly increased $\mathrm{S}$-phase population compared to that in the untreated control group (Fig. 5A and C). However, in NRF-1 overexpressing cells, BPDE treatment did not increase the proportion of cells in S-phase compared to the control vector, (Fig. 5C and D). These results indicated that upregulation of NRF-1 attenuated S-phase arrest induced by BPDE (Table II).

Subsequently, the effects of NRF-1 overexpression on cell cycle checkpoint activation in response to BPDE were assessed by western blot analysis. In cells transfected with control vector, BPDE treatment induced the activation of cell cycle checkpoint proteins ATM (Ser1981), Chk1 (Ser345),

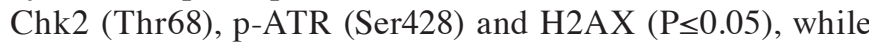
in cells transfected with NRF-1 overexpression plasmid, BPDE-induced activation of ATM, Chk2 and H2AX was significantly inhibited $(\mathrm{P}<0.05$ or $\mathrm{P}<0.01)$ (Fig. 6A and $\mathrm{B}$ ). However, the activation of Chk1 and ATR were not affected by NRF-1 overexpression plasmid compared to those in the cells transfected with control vector (Fig. 6A and C). These results suggested that NRF-1 can attenuate cell cycle arrest induced by BPDE via the regulation of ATM/Chk2 signaling.

\section{Discussion}

It is well known that BPDE-induces carcinogenesis by forming DNA adducts; these can be further degraded resulting in DNA strand breaks, which has been shown to induce cell cycle arrest (15). The $\mathrm{S}$ phase is considered the most genetically vulnerable phase of the cell cycle. Although BPDE and $\mathrm{BaP}$ have been reported to halt the cell cycle across multiple phases, which vary among different cell lines, the induction of S-phase arrest has been the most extensively studied $(16,17)$. A previous study by our group used $16 \mathrm{HBE}$ cells to investigate the apoptotic effects induced by BPDE and determined the involvement of mitochondrial dysfunction (14). The present study observed that treatment with BPDE caused a significant increase in the proportion of $16 \mathrm{HBE}$ cells in $\mathrm{S}$ phase, which was consistent with the results of Yao et al (17) using the same cell line.

The mechanisms of S-phase checkpoint arrest induced by BPDE may be explained from various viewpoints. The immunoblot data of the present study showed that the phosphorylation of Chk1 was increased in response to BPDE treatment, as well as its upstream DNA damage sensor ATR. The results were consistent with those of a previous study, which reported that BPDE induced ATR/Chk1 signaling and induced S-phase arrest (18). However, persistent blockage and the collapse of stalled forks may generate DNA double strand breaks (DSBs) that induce ATM/Chk2 signaling (12). It has been reported that complete cell cycle checkpoint arrest in response to ionizing radiation requires ATR as well as ATM reported (19). As expected, p-ATM and p-Chk2 were also involved in BPDE-induced S-phase arrest in the present study. In addition histone $\mathrm{H} 2 \mathrm{AX}$ was found to be phosphorylated at its $\mathrm{S} 139-\gamma$ position, which is a characteristic and commonly used marker for DSBs (20). Collectively, the results of the present study indicated that ATM/Chk2 and ATR/Chk1 signaling cascades may serve overlapping functions in order to cope with BPDE-induced DNA lesions, and the complexity of these interactions depends on the extent of DNA damage.

NRF-1 is one of several key transcription factors involved in nucleo-mitochondrial interactions and has a wide range of target genes (1). Together with other PGC-1 family co-activators, NRF-1 has a broad cyto-protective role in cellular physiology $(21,22)$. Aberrant regulation of NRF-1 and its targets has been indicated to contribute to the pathogenesis of human neurodegenerative diseases, diabetes mellitus and estrogen-dependent breast cancer $(6,23,24)$. Despite the fact that NRF-1 regulates metabolic processes, little is known with regard to its association with exogenous genotoxic stress. The present study observed that NRF-1 protein expression was suppressed by BPDE treatment. A previous study reported that NRF-1 was de-phosphorylated in quiescent BALB/3T3 fibroblasts and became phosphorylated upon cell cycle entry (25). In the present study, cells were treated with BPDE under low serum $(0.5 \%)$ conditions to minimize the impact of serum on NRF-1 phosphorylation. It was indicated that NRF-1 was suppressed by BPDE, possibly via direct phosphorylation by BPDE; however, it is possible that other kinases and cytokines may have mediated this process.

Flow cytometric analysis revealed that NRF-1 overexpression attenuated S-phase arrest induced by BPDE, but did not alter the cell cycle distribution in the absence of BPDE. The downregulation of basal NRF-1 may be an important event involved in BPDE-induced S-phase arrest. The present study determined that NRF-1 overexpression suppressed BPDE-induced phosphorylation of ATM and Chk2, while not affecting PBDE-induced increases in ATR and Chk1. Recently, Zambrano et al (26) reported that triiodothyronine (T3) induced DNA damage via ATM signal transduction. The activation of ATM was required for T3-dependent NRF-1 induction and oxidative stress. ATM-dependent mitochondrial biogenesis has been shown to be important in DNA damage response and regulation of reactive oxygen species (ROS) (27). Although NRF-1 appears to act downstream of ATM, the results of the present study suggested that NRF-1 overexpression may function via ATM/Chk2 signaling. It is therefore suggested that during the regulation of DNA damage, NRF-1 overexpression may be associated with retrograde signaling from the mitochondria to the nucleus.

A previous study by our group observed that ROS levels were increased following treatment with $\mathrm{BaP}$, the unmetabolized precursor of BPDE (14). Upregulation of NRF-1 is known to induce of the oxidative phosphorylation and anti-oxidant enzymes $(1,28)$. NRF-1 has also been reported to be involved in an anti-oxidant pro-survival network by controlling Tfam induction (21). Exogenous oxidative damage induces 
phosphorylation of NRF-1, which promotes the binding and activation of Tfam, thereby contributing to the maintenance of mtDNA and protection from oxidative damage (29). Whether overexpression of NRF-1 is able to reduce ROS formation and whether this mechanism contributes to the protective effects against BPDE-induced DNA damage remains to be validated.

In conclusion, the present study shed light on the mechanism of action of an environmental genotoxin and its effects on cell cycle checkpoints. It was demonstrated that BPDE triggers cell cycle arrest in S-phase by activating ATM/Chk2 and ATR/Chk1 signaling pathways. In addition, NRF-1 overexpression attenuated S-phase arrest induced by BPDE and suppressed the activation of ATM, Chk2 and H2AX. Although the exact role of NRF-1 in cell cycle regulation remains largely elusive, it may be hypothesized that NRF-1 overexpression is associated with BPDE-induced S phase arrest.

\section{Acknowledgements}

The authors would like to express their gratitude to Professor Weidong Zhu (Tongji University College of Medicine, Shanghai, China) for the assistance with the experimental design. In addition, the authors would like to thank Mr. Yangfei Li (Jiaotong University College of Medicine, Shanghai, China) and Dr Liang Zheng (Tongji University College of Medicine) for performing the data analysis. The present study was supported by the Nature Science Foundation of China (nos. 81001252 and 81170325) and the Shanghai Municipal Health Bureau Project (no. z0124y166).

\section{References}

1. Scarpulla RC: Nuclear control of respiratory chain expression by nuclear respiratory factors and PGC-1-related coactivator. Ann NY Acad Sci 1147: 321-334, 2008.

2. Gugneja S and Scarpulla RC: Serine phosphorylation within a concise amino-terminal domain in nuclear respiratory factor 1 enhances DNA binding. J Biol Chem 272: 18732-18739, 1997.

3. Elkon R, Linhart C, Sharan R, Shamir R and Shiloh Y: Genome-wide in silico identification of transcriptional regulators controlling the cell cycle in human cells. Genome Res 13: 773-780, 2003.

4. Cam H, Balciunaite E, Blais A, Spektor A, Scarpulla RC, Young R, Kluger Y and Dynlacht BD: A common set of gene regulatory networks links metabolism and growth inhibition. Mol Cell 16: 399-411, 2004.

5. Chiang MC, Cheng YC, Chen HM, Liang YJ and Yen $\mathrm{CH}$ : Rosiglitazone promotes neurite outgrowth and mitochondrial function in N2A cells via PPAR gamma pathway. Mitochondrion 14: 7-17, 2014.

6. Tong CW, Wang JL, Jiang MS, Hsu CH, Chang WT and Huang AM: Novel genes that mediate nuclear respiratory factor 1-regualted neurite outgrowth in neuroblastoma IMR-32 cells. Gene 515: 62-70, 2013.

7. Wallace DC: Mitochondria and cancer. Nat Rev Cancer 12: 685-698, 2012

8. Scarpulla RC: Transcriptional paradigms in mammalian mitochondrial biogenesis and function. Physiol Rev 88: 611-638, 2008.

9. Veatch JR, McMurray MA, Nelson ZW and Gottschling DE: Mitochondrial dysfunction leads to nuclear genome instability via an iron-sulfur cluster defect. Cell 137: 1247-1258, 2009.

10. Huo L and Scarpulla RC: Mitochondrial DNA instability and peri-implantation lethality associated with targeted disruption of nuclear respiratory factor 1 in mice. Mol Cell Biol 21: 644-654, 2001.
11. Eberhart J, Coffing SL, Anderson JN, Marcus C, Kalogeris TJ, Baird WM, Park SS and Gelboin HV: The time-dependent increase in the binding of benzo[a]pyrene to DNA through (+)-anti-benzo[a] pyrene-7,8-diol-9,10-epoxide in primary rat hepatocyte cultures results from induction of cytochrome P450IA1 by benzo[a]pyrene treatment. Carcinogenesis 13: 297-301, 1992.

12. Guo N, Faller DV and Vaziri C: Carcinogen-induced S-phase arrest is Chk1 mediated and caffeine sensitive. Cell Growth Differ 13: 77-86, 2002.

13. Mukherjee JJ and Kumar S: DNA synthesis inhibition in response to benzo[a]pyrene dihydrodiol epoxide is associated with attenuation of p(34)cdc2: Role of p53. Mutat Res 755: 61-67, 2013.

14. Sang H, Zhang L and Li J: Anti-benzopyrene-7,8-diol-9, 10 -epoxide induces apoptosis via mitochondrial pathway in human bronchiolar epithelium cells independent of the mitochondria permeability transition pore. Food Chem Toxicol 50: 2417-2423, 2012.

15. Venkatachalam $S$, Denissenko $M$ and Wani AA: DNA repair in human cells: Quantitative assessment of bulky anti-BPDE-DNA adducts by non-competitive immunoassays. Carcinogenesis 16 : 2029-2036, 1995.

16. Wang A, Gu J, Judson-Kremer K, Powell KL, Mistry H, Simhambhatla P, Aldaz CM, Gaddis S and MacLeod MC: Response of human mammary epithelial cells to DNA damage induced by BPDE: Involvement of novel regulatory pathways. Carcinogenesis 24: 225-234, 2003.

17. Yao B, Fu J, Hu E, Qi Y and Zhou Z: The Cdc25A is involved in S-phase checkpoint induced by benzo(a)pyrene. Toxicology 237: 210-217, 2007.

18. Bi X, Barkley LR, Slater DM, Tateishi S, Yamaizumi M, Ohmori H and Vaziri C: Rad18 regulates DNA polymerase kappa and is required for recovery from S-phase checkpoint-mediated arrest. Mol Cell Biol 26: 3527-3540, 2006.

19. Gatei M, Sloper K, Sorensen C, Syljuäsen R, Falck J, Hobson K, Savage K, Lukas J, Zhou BB, Bartek J and Khanna KK: Ataxia-telangiectasia-mutated (ATM) and NBS1-dependent phosphorylation of Chk1 on Ser-317 in response to ionizing radiation. J Biol Chem 278: 14806-14811, 2003.

20. Garcia-Canton C, Anadón A and Meredith C: $\gamma \mathrm{H} 2 \mathrm{AX}$ as a novel endpoint to detect DNA damage: Applications for the assessment of the in vitro genotoxicity of cigarette smoke. Toxicology In Vitro 26: 1075-1086, 2012.

21. Piantadosi CA and Suliman HB: Mitochondrial transcription factor A induction by redox activation of nuclear respiratory factor 1. J Biol Chem 281: 324-333, 2006.

22. Jones AW, Yao Z, Vicencio JM, Karkucinska-Wieckowska A and Szabadkai G: PGC-1 family coactivators and cell fate: Roles in cancer, neurodegeneration, cardiovascular disease and retrograde mitochondria-nucleus signalling. Mitochondrion 12: 86-99, 2012.

23. Liu Y, Niu N, Zhu X, Du T, Wang X, Chen D, Wu X and Gu HF: Genetic variation and association analyses of the nuclear respiratory factor 1 (nRF1) gene in Chinese patients with type 2 diabetes. Diabetes 57: 777-782, 2008.

24. Okoh V, Deoraj A and Roy D: Estrogen-induced reactive oxygen species-mediated signalings contribute to breast cancer. Biochim Biophys Acta 1815: 115-133, 2011.

25. Herzig RP, Scacco S and Scarpulla RC: Sequential serum-dependent activation of CREB and NRF-1 leads to enhanced mitochondrial respiration through the induction of cytochrome c. J Biol Chem 275: 13134-13141, 2000.

26. Zambrano A, García-Carpizo V, Gallardo ME, Villamuera R, Gómez-Ferreria MA, Pascual A, Buisine N, Sachs LM, Garesse R and Aranda A: The thyroid hormone receptor $\beta$ induces DNA damage and premature senescence. J Cell Biol 204: 129-146, 2014.

27. Valentin-Vega YA, Maclean KH, Tait-Mulder J, Milasta S, Steeves M, Dorsey FC, Cleveland JL, Green DR and Kastan MB: Mitochondrial dysfunction in ataxia-telangiectasia. Blood 119: 1490-1500, 2012

28. Patti ME, Butte AJ, Crunkhorn S, Cusi K, Berria R, Kashyap S, Miyazaki Y, Kohane I, Costello M, Saccone R, et al: Coordinated reduction of genes of oxidative metabolism in humans with insulin resistance and diabetes: Potential role of PGC1 and NRF1. Proc Natl Acad Sci USA 100: 8466-8471, 2003.

29. Suliman HB, Carraway MS, Tatro LG and Piantadosi CA: A new activating role for $\mathrm{CO}$ in cardiac mitochondrial biogenesis. J Cell Sci 120: 299-308, 2007. 